El presente artículo se propone tratar cómo la práctica reflexiva, un ARTICULOS · 149-162 aspecto que no se ha tenido demasiado en cuenta en los estudios más recientes sobre cómo se realiza el paso de novel a experto en interpretación, puede resultar clave en la formación de los futuros intérpretes y ser uno de los retos a los que debemos hacer frente en la actualidad. En ese sentido, y dada la importancia de fomentar tanto la conciencia como las competencias metacognitivas en el aula de interpretación, se presenta un ejemplo de instrumento adecuado a tal fin, la pauta metacognitiva. Se aborda su elaboración, así como el marco de aprendizaje más idóneo para incorporarla y se describe cómo trabajan con ella los estudiantes en el aula.

PALABRAS CLAVE: formación de intérpretes, autorregulación del aprendizaje, pauta metacognitiva, práctica reflexiva

\title{
Nuevos retos en la formación de intérpretes: la integración del componente metacognitivo en el aula
}

Marta Arumi Ribas

Universitat Autònoma de Barcelona
The article examines the role of reflective practice, an aspect which has been largely ignored in recent studies on the transition from novice to expert in interpreting training, as a potentially key factor in the training of future interpreters and one of the challenges which currently need to be addressed. In this regard, and given the importance of promoting awareness as well as metacognitive competencies in the interpreting classroom, an example of a tool appropriate for such development will be presented: the metacognitive guide. The development of this tool, as well as the learning framework best suited to its incorporation, is discussed. A description is also given of how students work with the proposed metacognitive guide in the classroom.

KEY WORDS: interpreting learning, self-regulation of learning, metacognitive guide, reflective practice 


\section{INTRODUCCIÓN}

Partiendo de las investigaciones más recientes en didáctica de la interpretación sobre cómo se realiza el paso de intérprete novel a experto, observamos cómo la práctica reflexiva, es decir, la introducción regular del componente reflexivo y del fomento de la metacognición en el aula, es un aspecto que no se ha tenido demasiado en cuenta en los distintos estudios.

Monereo apunta (1995: 75) que, históricamente, nuestra habilidad para explicitar las propias ideas y pensar en voz alta se ha utilizado en prácticas diversas, por ejemplo, para que los estudiantes se concienciaran de sus propios pensamientos y de su forma de actuar. El objetivo final es que los estudiantes puedan analizar y modificar sus pensamientos de manera autónoma, según cuáles sean sus necesidades.

La metacognición, o el conocimiento del conocimiento (Flavell, 200o), es una estrategia eficaz que permite al intérprete controlar su propia cognición con el objetivo de mejorar su rendimiento. $Y$ no únicamente en la fase profesional, sino también durante el proceso de formación. Ésta es la razón por la que los métodos de autoaprendizaje, autocontrol y autorregulación deberían tener una mayor presencia como parte integral de los currículos de formación de intérpretes. Los alumnos necesitan de una mayor transferencia de responsabilidad así como de un mayor grado de autonomía para poder seleccionar qué es lo más pertinente y apropiado para ellos en su proceso de aprendizaje.

En ese sentido, y dada la importancia de fomentar tanto la conciencia como las competencias metacognitivas en el aula de interpretación, presentamos un ejemplo concreto de propuesta didáctica adecuada a tal fin. El uso de instrumentos metacognitivos durante el proceso de aprendizaje de la interpretación puede ser un apoyo para la correcta adquisición de las habilidades necesarias para el desarrollo de la práctica interpretativa. La propuesta permite introducir la práctica reflexiva en el aula respondiendo a una doble finalidad:

a) Transferir paulatinamente a los estudiantes el control y la conciencia sobre las actividades de aprendizaje.

b) Fomentar una interacción que les ayude a tomar conciencia de todo lo relacionado con su propio proceso de aprendizaje.

\section{HACIA UN APRENDIZAJE CENTRADO EN EL ALUMNO}

Si observamos los planes de estudios tradicionales de las instituciones de formación en interpretación, tanto nacionales como extranjeras, apreciamos la existencia de cierto consenso sobre cómo se enseña la interpretación en las diversas modalidades (Mackintosh, 1995: 129). La mayoría de estos contenidos se fueron transmitiendo sin contrastar ni comprobar científicamente su eficacia (Gile, I995). La falta de estudios empíricos aplicados a estos postulados teóricos y didácticos contribuyó a que estos se fueran estableciendo como los únicos válidos. Así, la didáctica de la interpretación cuenta con una serie de ideas categóricas que subrayan la eficacia de ciertos ejercicios y su secuencia de aparición, y critican otros, sin que estas críticas estén fundamentadas en principios empíricos sólidos ( $I b$.).

No obstante, a partir de los años 90 del siglo pasado crece el interés por la didáctica, el cual se manifiesta en la publicación de diversas monografías (Gile, I995; Pöchhacker y Schlesinger 2002; Pöchhacker 2004) y en la creación de formaciones específicas para profesores de 
interpretación ${ }^{\mathrm{I}}$, normalmente intérpretes profesionales sin previa formación pero con un gran entusiasmo por transmitir y dar a conocer la profesión.

Ante los cambios sociales actuales, la adaptación de nuestra universidad al Espacio Europeo de Educación Superior, y la evolución de la profesión, se hace necesario un replanteamiento y una redefinición a fondo de la práctica pedagógica y la evolución hacia nuevos enfoques más sistemáticos y basados en los resultados de las investigaciones empíricas.

Uno de los grandes retos que vivimos hoy es el de contar con estudiantes cada vez más autónomos y participativos. Normalmente, cuando los estudiantes acceden a la formación, suelen presuponer que el aprendizaje tendrá lugar mayoritariamente dentro de las cuatro paredes del aula. Por esta razón, hay que esforzarse por despertar en ellos aquellas habilidades que les permitan describir su experiencia de aprendizaje, planificarla y monitorizarla, y disponer del metalenguaje necesario para poder analizar y autoevaluar sus prestaciones.

Según Little (1997), no puede haber autonomía sin una implicación consciente en el aprendizaje, ya que la finalidad básica consiste en preparar a los alumnos para un aprendizaje «de por vida». La autonomía, en este sentido, se entiende como la capacidad de tomar decisiones, actuar de forma independiente y reflexionar de manera crítica y objetiva. La reflexión consciente incluye tanto los conocimientos y saberes como los procesos necesarios para adquirirlos y ha de conducir necesariamente a un proceso de autorregulación, sin el cual es imposible alcanzar el aprendizaje de por vida (Rampillon, 1997).

\footnotetext{
I Destacamos el Master of Advanced Studies for Interpreter Trainers de la École de Traduction et Intérpretation de la Universidad de Ginebra.
}

Como docentes, se nos plantea de inmediato la cuestión de cuál es la acción o las acciones pedagógicas que mejor fomentan la autonomía. Encontramos respuesta en los principios de la teoría sociocultural del aprendizaje (Lantolf, 2000), la cual postula que el aprendiz construye nuevos significados y conocimientos mediante la interacción, porque es a través de ésta que se fomentan procesos cognitivos superiores que favorecen el aprendizaje.

Apunta Moser-Mercer (2005a: 65) que para que los estudiantes puedan situarse en su proceso de aprendizaje y considerar el aprendizaje fuera del aula como parte intrínseca de la experiencia en general, hay que disponer de una buena comprensión de cómo funciona el proceso de interpretación. Es imprescindible tener una buena división del proceso en habilidades y subhabilidades, y contar con una definición muy detallada de la progresión necesaria.

\section{EL DESARROLLO DE LA PERICIA: EL PASO DE INTÉRPRETE NOVEL A EXPERTO}

En el campo de la didáctica de la interpretación, se han elaborado varios estudios que definen cómo se produce el paso de intérprete novel a experto. Hoffman (I997b) y MoserMercer et al. (2000) subrayan que el desarrollo de la pericia tiene lugar en distintas fases, que suponen una progresión desde una comprensión superficial y literal de los problemas hasta una comprensión más articulada y conceptual. Anderson (1995: 2I) describe tres fases en concreto para la adquisición de las distintas habilidades:

a) la fase cognitiva: durante la cual los principiantes desarrollan un conocimiento declarativo de la habilidad;

b) la fase asociativa: donde los principiantes van detectando y eliminando sus errores. A su

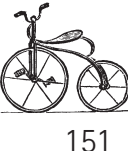

151 
vez, establecen conexiones entre los distintos elementos, necesarios para un buen dominio de la habilidad;

152 c) la fase autónoma: en la que los procedimientos cada vez son más automáticos.

Ericsson (200I: 193) subraya que la adquisición de la pericia requiere de práctica deliberada. No se trata de la práctica por la práctica, sino de la que se realiza con un alto grado de motivación, la que se sitúa en tareas bien definidas, con retroalimentación y oportunidades de repetición. En este sentido, se trata de la que tiene como objetivo la mejora del rendimiento. El concepto de práctica deliberada destaca la importancia que tiene el hecho de ayudar a los alumnos a controlar su aprendizaje, a buscar retroalimentación que les permita evaluar sus estrategias y niveles. Y Ericsson (ib. 194) apunta la importancia de los formadores, ya que desempeñan un papel decisivo a la hora de guiar a los futuros expertos. Así el profesor deberá crear el andamiaje que permita que los estudiantes avancen hacia un nivel de desarrollo superior a su nivel actual a través del planteamiento de retos cognitivos viables.

Según Bransford et al. (2000), el experto es aquel que ha adquirido conocimientos sobre el contenido, que se organizan de manera que pueda reflejar una comprensión profunda del tema. Asimismo, el conocimiento del experto no se puede reducir a un conjunto de hechos aislados, sino que debe reflejar los contextos de aplicación y es suficientemente flexible para recuperar con flexibilidad aspectos importantes de su conocimiento con poco esfuerzo de atención.

En esta línea, existen dos tipos de expertos: los expertos rutinarios y los expertos con capacidad de adaptación. Según Hatano e Inagaki (1986) los expertos con capacidades de adaptarse son aquellos que se integran con mayor faci- lidad a nuevas situaciones. Son metacognitivos y no pretenden únicamente ser más eficaces en su ejecución de las tareas, sino hacerlo en un nivel superior.

\section{LA IMPORTANCIA DE LA PRÁCTICA REFLEXIVA Y LA AUTORREGULACIÓN EN LA FORMACIÓN DE INTÉR PRETES}

Algunos autores se han referido a la relación existente entre las prácticas metacognitivas y autorreguladoras y la formación de intérpretes (Ficchi, 1999). Así, el desarrollo del pensamiento metacognitivo parece ir en paralelo con el tipo de razonamiento que conduce a la pericia o dominio de un campo. Moser-Mercer et al. (2000: IIO) aportan algunos elementos sobre cómo se produce el salto de un nivel a otro:

In interpreting, novices need to engage in tactical learning whereby they learn specific rules for solving specific problems, such as how to convert particular syntactic constructions in the incoming message to matching constructions in the outgoing language. This tactical knowledge then becomes increasingly well organized and the novice develops a set of strategies (monitoring strategies, workload management strategies, etc.) designed to optimally solve the problems he encounters.

Seguramente, «el aprendizaje táctico» y «el conjunto de estrategias» a los que se refieren los autores se pueden reforzar a través de las herramientas metacognitivas que fomentan la eficacia en el aprendizaje y que allanan el paso de un nivel a otro. En este sentido, la conciencia sobre el aprendizaje debería constituir la base de la formación en interpretación.

Según Perry et al. (2006), los estudiantes que se autorregulan consiguen hacer un buen uso de las oportunidades que se les presentan, afrontan los retos de aprendizaje y práctica, desarrollan una 
comprensión profunda del tema y se esfuerzan para conseguir sus objetivos. En entornos de estas características, los estudiantes suelen encontrarse con dificultades al comienzo, a la hora de gestionar su aprendizaje, ya que están más acostumbrados a entornos de aula más convencionales y centrados en el profesor (Sainz, 1997: I35). La posición del profesor en clase debería cambiar de alguna manera y, como apunta Kiraly (2000: 33), pasar de una perspectiva «transmisionista», en la cual transmite los conocimientos y los estudiantes trabajan solos, a una perspectiva «transformadora», en la cual el aprendizaje constituye un proceso de construcción donde profesores y estudiantes colaboran en una «comunidad de construcción de conocimiento".

Otra cuestión que argumenta Moser-Mercer (2005a) es el hecho de que la mayoría de los métodos pedagógicos en interpretación se centran en crear lo que hemos descrito como expertos rutinarios, es decir, expertos que pueden integrarse bien en un gran número de situaciones parecidas y repetidas. La autora apunta que, con la introducción de nuevas formas de interpretación, cada vez más el intérprete tiene que hacer frente a situaciones nuevas e incluso inesperadas. Y es así como se plantea la cuestión de cómo podemos ir más allá de los principios pedagógicos actuales y conseguir, a través de la enseñanza-aprendizaje, la emergencia de profesionales que sean capaces de adaptarse a nuevos entornos sin dificultad. Se trata de crear entornos y situaciones que fomenten el aprendizaje metacognitivo, es decir, contextos y prácticas de aprendizaje que subrayen cómo se puede organizar el conocimiento y qué capacidades estratégicas son necesarias para resolver los problemas que emergen.

A partir de esta reflexión, nace la propuesta didáctica que describimos en los próximos apartados, con el objetivo de encontrar un instrumento que permita a los estudiantes que se inician en la interpretación consecutiva reflexionar sobre su progreso en el aprendizaje de esta modalidad.

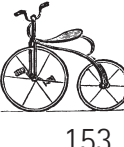

153

\section{DESCRIPCIÓN DE LA PROPUESTA DIDÁCTICA}

La experiencia docente nos revela que los estudiantes suelen tener interés por la interpretación y que suelen practicar por su cuenta pero que, sin ayuda externa, no suelen realizar el siguiente paso, es decir, analizar de forma activa y sistemática su rendimiento. Esperábamos que esta propuesta les permitiese identificar conductas y estrategias, hacer correcciones y tomar la distancia objetiva necesaria. También esperábamos que sirviese como instrumento que pudiera llegar a impulsar y a desencadenar el cambio.

\section{I. ¿Qué son las pautas metacognitivas?}

No es que pensemos que el aprendizaje de la interpretación consecutiva no se puede dar sin recurrir a propuestas de esta índole. Lo que sí defendemos es que, con la integración de instrumentos metacognitivos, el aprendizaje es más consciente, ya que estos permiten documentar el proceso, tener evidencias sobre cada uno de los pasos y aprender a hacer una lectura crítica y a interpretarlos.

La acción pedagógica que describiremos seguidamente consiste en la introducción regular, en el aula de interpretación consecutiva, de unos instrumentos, las pautas metacognitivas, con los que trabajarán los alumnos periódicamente.

Desde el ámbito de la Didáctica y de la Psicología de la Educación, no existe una única denominación consensuada del instrumento 
que para Monereo (I995: 78) es una herramienta de la que dispone el profesor para transferir el control de cada actividad a los estudiantes favoreciendo así que interioricen o se apropien del significado de los contenidos curriculares tratados para que puedan llegar a utilizarlos de manera independiente. En la misma línea, en Arumí (2003: I9I) se define como una guía escrita pensada para que el alumno la utilice para la reflexión y la explicitación sobre su proceso de aprendizaje individual o respecto a una habilidad concreta.

Monereo et al. (1998) consideran que los sistemas de enseñanza por interrogación consisten en un conjunto de cuestiones que inciden en las principales variables de un proceso de aprendizaje ligado a una tarea, de manera que la respuesta que reclaman facilita su resolución. Esto se refleja en el hecho de que, cuando estos interrogantes se centran en las acciones mentales o en los procesos que hay detrás de estas acciones, hablamos de interrogación cognitiva y metacognitiva.

Según Monereo et al. (I998), existen dos grandes modalidades que habitualmente se utilizan de forma complementaria: la interrogación externa que formula el profesor como si se tratase de una ayuda con el fin de comprender y responder mejor a la materia, y la autorregulación que el propio alumno se administra en calidad de guía interna que le permite controlar y mejorar su aprendizaje. En la práctica, afirma, la primera suele ir antes y provocar la segunda favoreciendo así la apropiación gradual y la automatización de los interrogantes por parte del alumno en el camino hacia su emancipación cognitiva.

Por otro lado, este autor ve en las guías de interrogación una forma mediante la cual el profesor puede ofrecer modelos de interrogación que facilitan al aprendiz la reflexión sobre su proceso cognitivo. La finalidad de estos modelos es que, cuando posteriormente los alumnos trabajen de manera independiente, sean capaces de elaborar sus propias guías que les permitan reflexionar sobre sus decisiones.

Respecto a nuestra propuesta concreta para el aprendizaje de la interpretación consecutiva, entendemos las pautas metacognitivas como cuestionarios $^{2}$, en forma de preguntas o afirmaciones, cuya función consiste en guiar la reflexión del alumno para que explicite su propio aprendizaje. Se trata de un cuestionario que tiene que ser lo más sencillo y concreto posible y que tiene que llevar al alumno, a través del «desmenuzamiento previo del proceso», a formularse explícitamente las características de la actividad de la interpretación consecutiva. El alumno tiene que ser capaz de reconstruir retroactivamente el proceso cognitivo que debe seguir al realizar una tarea concreta de interpretación ${ }^{3}$.

El objetivo del trabajo con pautas metacognitivas es que el aprendiz, de forma continuada, vaya reflexionando por escrito sobre algunos o todos los puntos que contiene la pauta. Main (I985) afirma que los métodos más productivos para la mejora del aprendizaje son aquellos en los que se estimula a los alumnos a reflexionar de manera crítica sobre sus propias actuaciones. Queremos que la pauta dé pie a un proceso dialógico, del aprendiz consigo mismo, pero también del aprendiz con el profesor y con

\footnotetext{
Anexo I.

2 Véase un ejemplo de pauta metacognitiva en el

3 En este artículo presentamos únicamente el valor de la pauta como instrumento didáctico. E1 lector puede encontrar su aplicación como herramienta de investigación en Arumí y Esteve (2006). También en Abuín (2007) se ofrece una amplia panorámica de los métodos retrospectivos que se han utilizado en la investigación sobre el proceso de interpretación.
} 
sus compañeros. La pauta es la forma como el docente guía la reflexión sobre la tarea y la manera como los aprendices dan también información al docente, que después podrá hacer los reajustes necesarios a partir de la información recibida. Aunque las pautas son un encargo del docente, tal y como apunta Bakhtin (I98I), cuando escribimos o hablamos, dirigiéndonos a otra persona, lo que estamos haciendo también es hablar con nosotros mismos. Y verbalizar contribuye a la clarificación de las ideas.

Las pautas metacognitivas contemplan los componentes básicos de conciencia y control propios de todo proceso de autorregulación. Tal y como apunta Van Lier (1996), para aprender es absolutamente esencial que haya una implicación consciente en el aprendizaje, y eso no implica sólo una atención específica en los contenidos, sino también conocer todos los procesos que tienen lugar durante el aprendizaje, de lo que pasa y de lo que se está haciendo, en definitiva, la implicación de una «mente consciente». Así como hay estudios que han llegado a la conclusión de que la conciencia lingüística es esencial en el aprendizaje de lenguas extranjeras por parte de adultos, también es importante que los estudiantes desarrollen lo que podríamos denominar la conciencia interpretativa. Y una forma de hacerlo es a partir de metodologías que incorporan y prevén fases donde el aprendiz se ve obligado a reflexionar (individualmente o con otros) sobre diversos aspectos relacionados con la tarea que está llevando a cabo.

Al mismo tiempo, la pauta es un instrumento que permite transferir al aprendiz, paso a paso y de forma sistemática, no el control de una actividad global, sino el control de los procesos cognitivos o de las operaciones mentales que tienen lugar en una tarea relacionada con una habilidad concreta.
Los objetivos concretos del trabajo con pautas metacognitivas son:

-Que, dentro de la globalidad del proceso,

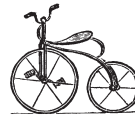

155 los aprendices puedan diferenciar y, por tanto, reflexionar sobre cada una de las fases de la interpretación consecutiva.

-Que los aprendices puedan verbalizar sus reflexiones sobre el aprendizaje.

-Que cada uno pueda apreciar cuál es su progreso y también todos los aspectos en los que no se han obtenido los resultados esperados.

-Que se puedan centrar en las carencias y en los errores.

- Reforzar en el alumno los aspectos que domina y pedirle la explicitación de las estrategias que considera positivas.

-Que la pauta pueda ser también un punto de referencia en el momento de tomar las diversas decisiones por las que hace falta optar en el ejercicio de la interpretación.

-Que se pueda hacer un seguimiento dinámico del proceso de aprendizaje porque cubren un período largo de tiempo.

\subsection{La elaboración de las pautas metacognitivas y su aplicación}

Queremos ahora centrarnos en los elementos que, según Ribas (2000), permiten describir el funcionamiento y la utilidad de las pautas. Esta autora señala que la realidad de las prácticas educativas proporciona una gran diversidad de pautas y un abanico muy amplio de maneras de utilizarlas.

En la acción pedagógica que nos ocupa, las pautas metacognitivas se refieren a dos objetos diferenciados. Por un lado, cada una de las habilidades por separado que hace falta dominar 
en un ejercicio de interpretación consecutiva ${ }^{4} y$, por otro, el proceso global de aprendizaje de las habilidades anteriores. En cuanto al grado de generalización, las pautas con las que trabajamos son bastante generales para que se puedan aplicar a todas las actividades de interpretación que realizan habitualmente los alumnos en clase.

En relación con las formas de utilización, los aprendices han hecho un uso sólo individual, si bien es cierto que, en otras ocasiones, las pautas pueden servir como la base del trabajo de discusión en parejas o en grupo.

Se pueden introducir en diversos momentos del proceso de aprendizaje y con objetivos particulares múltiples. En este caso, los estudiantes han trabajado siempre después de haber hecho una tarea de interpretación consecutiva con el fin de que les sirviesen para hacer un ejercicio de retrospección del proceso seguido y de valoración del discurso resultante.

Con respecto a su elaboración, el docente la puede hacer previamente y después darles las pautas para que trabajen o bien la elaboración puede ser conjunta, entre docente y aprendices, como tarea de clase. En nuestro caso, las pautas fueron elaboradas por la profesora.

Como apunta Ribas (2000), la presentación y el formato de la pauta pueden conducir al alumno hacia diferentes tipos de respuesta. Hay diversidad de pautas, desde las que no interactúan de manera explícita con el lector hasta las que llevan a la acción, pasando por las que dialogan con el alumno. En cuanto a la orientación de la respuesta, algunas refuerzan los aspectos que el alumno

4 No existe un consenso sobre el número de fases en las cuales se descompone el proceso de interpretación consecutiva (Bowen y Bowen, I984: 3; Weber, I989: I63 Gile, I995: I78 y Seleskovitch y Lederer, I989). Ante esta diversidad de opiniones en cuanto a la segmentación del proceso de la consecutiva, hemos optado por no basarnos en una propuesta única sino en incorporar ciertos elementos de todas las existentes. domina, pidiendo explicitar las estrategias que considera positivas. Otras, en cambio, ponen más énfasis en las carencias o errores.

\section{3. ¿¿Cuál es el marco de aprendizaje más idóneo para el trabajo con pautas metacognitivas y cómo debe ser la intervención del docente?}

Una acción pedagógica de estas características no puede presentarse de forma aislada sino como parte integrante de una globalidad y de un marco de aprendizaje. En este sentido, partimos de la teoría de Galperin (1992), que describe el aprendizaje como una actividad orientada hacia el objeto, como un proceso en el cual la acción pasa por distintas fases. Concretamente, subraya la importancia de la existencia de cuatro fases en el proceso de enseñanza-aprendizaje: I) la orientación; 2) el pensamiento comunicativo; 3) el pensamiento dialógico; y 4) la actuación mental. Estas cuatro fases se pueden contemplar en una secuencia didáctica que parta de la orientación, es decir, que promueva, con las primeras actividades, que el estudiante se sitúe en su Zona de Desarrollo Próximo (ZDP) 5 respecto al objetivo $u$ objetivos concretos de aprendizaje a alcanzar. La segunda fase de la secuencia consistiría en lo que Galperin denomina pensamiento comunicativo y dialógico. Las actividades reflexivas (individuales o en grupo), desencadenadas por la incorporación del trabajo con la pauta, promueven la interacción con los otros y con uno mismo. Y es así como se verbalizan los procesos cognitivos y

5 Vygotsky (I978) define la Zona de Desarrollo Próximo como el estadio cognitivo del alumno que puede transformarse a partir de la interacción con otros. Según los presupuestos vygotskianos, durante el aprendizaje se constatan dos niveles de desarrollo: uno actual que representa aquello que el alumno sabe y sabe hacer por sí mismo, y otro potencial, que representa lo que el alumno puede ser capaz de hacer a partir de la ayuda que recibe de otros. 
metacognitivos. Por último, la fase final de este proceso consistiría en el momento en el cual la acción se ha integrado de tal forma que se ha convertido en un acto mental. En este sentido, el aprendiz puede aplicar lo aprendido a situaciones similares a partir de la experiencia adquirida.

Para el trabajo con pautas metacognitivas hace falta tener en cuenta las siguientes consideraciones:

-Que las pautas se integren, lo máximo posible, en la actividad puntual del alumno en el ámbito de marcos u objetivos más amplios en los que la pauta puede adquirir significado de manera adecuada.

- Posibilitar la participación de todos los alumnos en la elaboración de las pautas.

-Que los alumnos trabajen conjuntamente con los resultados obtenidos individualmente con las pautas, o bien que trabajen en grupo con una misma pauta.

-Utilizarlas periódicamente para que, con el tiempo, lleguen a formar parte de los materiales del alumno y éste las utilice regularmente.

-Incluirlas en el currículo, tanto en el externo, que establece la programación, como en el interno, es decir, hacerlas aparecer o mencionarlas en todas las situaciones que se van dando, también en lo que no está planificado.

- Promover, cuando ya hace un tiempo que se trabaja con pautas, el uso autónomo de éstas.

-Integrarlas en un entorno de aprendizaje basado en el fomento de los principios socioconstructivistas de la interacción y co-construcción de conocimientos y del fomento de la autonomía. En una docencia en la cual las representaciones y los conocimientos interiorizados de los aprendices ocupen un lugar privilegiado.

-A través de las pautas el docente está trabajando en las ZDP de los alumnos y les está ofreciendo ayuda pedagógica (Onrubia, I993). Por tanto, debe tener en cuenta los esquemas de conocimientos de los alumnos en relación con el contenido de aprendizaje tratado y tomar como punto de partida los significados y los sentidos de los que disponen los alumnos en relación

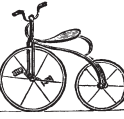

157 con aquellos contenidos. Al mismo tiempo, tiene que provocar retos que lleven al alumno a cuestionarse estos significados y sentidos con el objetivo de modificarlos cuando sea necesario.

- Si bien las pautas tienen que estar muy guiadas al inicio, el objetivo es que gradualmente esta guía pueda ir desapareciendo hasta que se pueda llegar a un momento en el que se pueda prescindir de ellas y se puedan retirar.

-El profesor puede utilizar el trabajo con las pautas como base para las tutorías ${ }^{6}$. Una vez que los estudiantes han reflexionado en las pautas sobre las tareas realizadas, necesitan, como mínimo durante el primer tiempo de familiarización con el instrumento, a alguien que corrobore y que guíe si las reflexiones llevadas a cabo son o no las acertadas. Necesitan la intervención externa de alguien más experto que les ayude a planificar cómo avanzar a partir de todo lo que han detectado.

\section{4. ¿Cómo trabajan con pautas metacognitivas los estudiantes en el aula de interpretación consecutiva?}

La reflexión de los aprendices en las pautas se plasma por escrito ${ }^{7}$. El discurso escrito permite que tanto el grupo como el individuo progresen en la construcción de conocimiento. Un texto escrito, a diferencia del texto oral, es un artefacto permanente que se puede revisar, repensar a través de una forma diferente de diálogo, en el que el texto que se está construyendo juega un papel

6 Las tutorías deben servir como un encuentro de orientación del estudiante y son, a nuestro entender, una actividad esencial que acompaña el proceso formativo.

7 Véase un ejemplo del trabajo de un estudiante en el anexo 2. Se trata de la reflexión sobre su habilidad en la toma de notas, pocos días después de que esta técnica haya sido introducida en clase. 
central. E1 texto funciona como un objeto que proporciona el tema central del discurso progresivo y, al mismo tiempo, incorpora simultáneamente el progreso que se ha alcanzado. Existe una unión estrecha entre escribir y aprender. A través de la escritura uno ordena sus ideas, las relaciona, las modifica y genera nuevas.

En el caso que nos ocupa, durante la primera mitad del semestre, aproximadamente, se introducen seis pautas, una diferente cada semana. Cada una de ellas está orientada hacia las competencias o habilidades que es fundamental dominar para la realización de un buen ejercicio de interpretación consecutiva:

Pauta $I$ : escucha, atención y concentración;

Pauta 2: memoria y análisis del discurso original;

Pauta 3 : toma de notas;

Pauta 4: comprensión del discurso en la lengua extranjera;

Pauta 5: reproducción y presentación del discurso en la lengua meta;

Pauta 6: pauta global que contempla cada una de las habilidades anteriores.

Al mismo tiempo, para cada una de las habilidades, las pautas incorporan un desglose minucioso de subhabilidades que tiene que permitir que el aprendiz llegue a reflexionar sobre el nivel más detallado de su actividad cognitiva. A continuación, se introduce una única pauta que engloba todas las competencias anteriores y que los aprendices utilizan durante diversas semanas consecutivas. Los alumnos trabajan con las pautas de forma retrospectiva en clase, habitualmente con una producción oral al lado.

\section{CONSIDERACIONES FINALES}

La integración de instrumentos y estrategias metacognitivas en la formación es un enfoque pedagógico que permite concentrarse más en el proceso, e invita a un aprendizaje más autónomo y más centrado en el aprendiz. A su vez, potencia que los estudiantes se responsabilicen de su propio aprendizaje. Los estudiantes no deben conocer únicamente los objetivos generales, sino también las competencias y habilidades específicas que deben dominar en cada momento. Sólo así sabrán en qué fijar su atención, con la ayuda de los instrumentos metacognitivos, que actúan de guía en todo este proceso.

El uso regular de la metodología presentada, en las clases de interpretación consecutiva, nos permite extraer algunas conclusiones sobre la eficacia de este método. Las pautas, a través de la verbalización de la reflexión sobre el propio aprendizaje, permiten a los estudiantes:

-Autorregular el proceso de aprendizaje de la interpretación consecutiva, es decir, tomar conciencia y controlarlo a través de la evaluación, la monitorización y la planificación;

-Establecer relaciones con el contexto de aprendizaje: con los profesores, con el resto de compañeros y con el entorno;

- Expresar cuestiones afectivas;

-Integrar, en la verbalización, el metalenguaje y la información teórica propios de la disciplina.

Observamos, a su vez, que la pauta metacognitiva es una buena guía para la reflexión introspectiva de los aprendices. Su función puede variar durante el transcurso de una asignatura. Al comienzo, como los estudiantes no están acostumbrados a su uso, les ayuda a tener una representación de qué se espera de ellos y a anticipar qué conocimientos serán necesarios para realizar un buen ejercicio de introspección. A medida que avanza el curso, y con la experiencia adquirida, la pauta fomenta más la retrospección sobre el proceso seguido y la evaluación del producto obtenido. Asimismo, a medida que avanza el aprendizaje, los aprendices adquieren 
mayor seguridad y autonomía. Poco a poco van alejándose del contenido y del guiaje específicos de la pauta para hacer una reflexión más libre y adaptada a las necesidades emergentes. También la pauta contribuye a relacionar el proceso de la interpretación con el del aprendizaje del alumno. A través de la explicitación del proceso, los estudiantes otorgan una representación a los contenidos del aprendizaje y al control sobre el propio proceso. Cabe destacar también que la pauta contribuye a que la reflexión sobre el proceso sea más detallada, precisa y sistemática, en tanto que la guía y la relaciona directamente con una tarea cognitiva concreta.

Un enfoque como el que hemos descrito conduce inevitablemente a un replanteamiento de las funciones de docente y aprendiz, de los objetivos y de las técnicas, y fomenta la adopción de una nueva perspectiva en cuanto a la metodología y a la función y naturaleza de la evaluación.

Aunque nuestra reflexión se ha centrado en la modalidad presencial del aprendizaje, no queremos terminar sin referirnos brevemente a las nuevas modalidades emergentes de aprendizaje. Dadas las ventajas que aportan los entornos de aprendizaje virtual, que ya se están estableciendo en diversos campos, parece probable que en un futuro no demasiado lejano existan programas de formación de intérpretes a distancia. Existen ya algunos centros de formación de intérpretes, como la École de Traduction et Interpretation de Ginebra (Moser, Class \& Seeber, 2004; Motta, 2006) o la Copenhagen Business School que han desarrollado algunos complementos formativos a distancia. Y, evidentemente, en entornos de trabajo menos mediados por el profesor y en los cuales el trabajo autónomo e individual gana importancia, las herramientas metacognitivas serán cruciales para guiar y acompañar el proceso del estudiante en su experiencia de aprendizaje.

RECIBIDO EN DICIEMBRE 2007 ACEPTADO EN MAYO 2008

\section{ANEXO I. Ejemplo de pauta metacognitiva}

\section{PAUTA DE REFLEXIÓN 3 SOBRE LA TOMA DE NOTAS}

\section{Reflexiona sobre los siguientes aspectos y contesta:}

I. ¿En qué lengua tomo las notas? ¿Me es fácil encontrar la traducción de los términos en el caso de que las tome en la lengua meta?

2. ¿Mi toma de notas es suficientemente clara?

3. ¿Considero que escribo demasiado?

4. ¿Utilizo símbolos y abreviaturas? ¿Cómo consigo interiorizar los símbolos e ir desarrollando un sistema de símbolos propio?

5. ¿Podría tomar las notas de forma más concisa?

6. ¿Establezco una buena relación entre las ideas? ¿Utilizo conectores?

7. Después de escuchar el discurso original y tomar notas, suelo disponer de unos segundos antes de ponerme a hacer la interpretación del discurso. Con un simple vistazo a mis notas, ¿consigo tener una idea clara de la estructura del discurso, de las ideas principales y secundarias, causas y consecuencias, etc.?

8. ¿Deseo añadir alguna reflexión que no ha aparecido en las preguntas anteriores? 
ANEXO 2. Ejemplo del resultado del trabajo de un estudiante con la pauta metacognitiva anterior

I. ¿En qué lengua tomo las notas? ¿Me es fácil encontrar la traducción de los términos en el caso de que las tome en la lengua meta?
TEXTO DE LA PAUTA

\section{TEXTO ESTUDIANTE}

Esta pregunta es difícil de responder porque todavía no hemos practicado discursos que tengamos que interpretar de una lengua extranjera, el alemán en nuestro caso, a nuestra lengua materna, así que no sabría qué contestar. En principio, sí he anotado un símbolo, supongo que podría servir para varios idiomas.

Como no siempre encuentro un símbolo en el momento oportuno, si tengo que escribir una palabra de manera explícita, lo hago en castellano (pero, como he mencionado, el discurso de partida también está en este idioma).

No suelo utilizar palabras inglesas porque no es una lengua muy cercana aunque sí utilizo siempre «hi!» (=saludar, quiero saludar, me gustaría saludar) y «thanks» (= agradecer, agradezco, quiero agradecer, me gustaría agradecer). En todo caso, «hi!» más que del inglés, sería extraída del alemán (ahora que lo pienso).

2. ¿Mi toma de notas es suficientemente clara?

Mi toma de notas es un poco caótica y casi «sucia» pero no se debe a que yo sea muy desordenada sino a que tengo una manera muy extraña de colocar el folio al escribir. Con la toma de notas tengo que poner el cuaderno recto y, claro, en vez de «escribir» en línea recta, se me inclina la dirección.

$3 \cdot$ ¿Considero que escribo demasiado?

No considero que escriba mucho; es más, a veces, por no haberlo querido escribir explícitamente, si no se me ha ocurrido algún símbolo, me quedo con menos información que quizás después me falta para detallar más.

4. ¿Utilizo símbolos y abreviaturas? ¿Cómo consigo interiorizar los símbolos e ir desarrollando un sistema de símbolos propio?

Intento utilizar el máximo de símbolos posibles porque desde que empezamos me quedé con la frase «mejor que escribáis poco y con símbolos a escribirlo todo con palabras» (más o menos).

Creo que la única solución para interiorizarlos es la práctica.

Además de esto, quizás el trabajo en grupo porque se pueden comentar las diferentes opciones para un mismo símbolo y adoptar la solución de alguna compañera que no se nos había ocurrido antes.

5. ¿Podría tomar las notas de forma más concisa?

Seguro que sí pero cuando domine más la toma de notas y asimile bien y amplíe el listado de símbolos propios.

6. ¿Establezco una buena relación entre las ideas? ¿Utilizo conectores?
La primera toma de notas, si no recuerdo mal, fue un desastre en cuanto a lo que conectores se refiere. Ahora cada vez intento incorporarlos más y marcar la separación de ideas claramente porque me doy cuenta de que sin ella no sabía donde empezaba o acababa la frase y si los símbolos de arriba se enlazaban o no con los de más abajo. 


\begin{tabular}{|c|c|}
\hline $\begin{array}{l}\text { 7. Después de escuchar el dis- } \\
\text { curso original y tomar notas, } \\
\text { suelo disponer de unos segun- } \\
\text { dos antes de ponerme a hacer } \\
\text { la interpretación del discurso. } \\
\text { Con un simple vistazo a mis } \\
\text { notas, ¿consigo tener una idea } \\
\text { clara de la estructura del dis- } \\
\text { curso, de las ideas principales } \\
\text { y secundarias, causas y conse- } \\
\text { cuencias, etc.? }\end{array}$ & $\begin{array}{l}\text { No, todavía no he llegado a ese nivel tan perfecto. Quizás en algunos } \\
\text { fragmentos sí que puedo reconocer todo de manera más o menos clara } \\
\text { pero, en general, en el total, aún no. Supongo que tengo que conse- } \\
\text { guir, primero, una toma de notas ordenada y clara, no dejarme ningún } \\
\text { conector ... }\end{array}$ \\
\hline $\begin{array}{l}\text { 8. ¿Deseo añadir alguna } \\
\text { reflexión que no ha aparecido } \\
\text { en las preguntas anteriores? }\end{array}$ & $\begin{array}{l}\text { Simplemente quiero aclarar que en la toma de notas que tenemos } \\
\text { que adjuntar puede haber alguna modificación o mejora que hayamos } \\
\text { comentado, suelen estar en otro color o a la derecha de la columna. }\end{array}$ \\
\hline
\end{tabular}

\section{REFERENCIAS BIBLIOGRÁFICAS}

Abuín, M. (2007). El proceso de interpretación consecutiva. Un estudio del binomio problemalestrategia. Granada: Comares.

Anderson, J. R. (1995). Cognitive Psychology and its Implications. Nueva York: W. H. Freeman.

Arumí, M. (2003). La reflexió metacognitiva en els nivells inicials de l'ensenyament i l'aprenentatge de la interpretació consecutiva en l'àmbit universitari: un estudi etnogràfic. Trabajo de investigación inédito. Departamento de Traducción y Filología de la Universitat Pompeu Fabra. (2006). Incidència d'una acció pedagògica dirigida a l'autoregulació. Dos estudis de cas a l'aula d'iniciació a l'aprenentatge de la interpretació consecutiva. Tesis doctoral inédita. Departamento de Traducción y Filología. Universitat Pompeu Fabra.

Arumí, M. y O. Esteve (2006). «Using instruments aimed at self-regulation in the foreign language and consecutive interpreting classrooms: An ethnographic-ecological approach to research». Electronic Journal of Foreign Language Teaching, 3/2, pp. I58-189.

Bakhtin, M. M. (I98I). The Dialogic Imagination, Four Essays. Editado por M. Holquist. Austin TX: University of Texas Press.
Bowen, D. y M. Bowen (1984). Steps to Consecutive Interpretation. Washington, D. C.: Pen and Booth.

Bransford, J.; Brown, A. L.; \& Cocking, R. R. (eds.) (2000). How people learn. Brain, mind, experience and school. Washington, D. C.: The National Academic Press.

Ericsson, K. A. (200I). «Expertise in interpreting. An expert-performance perspective». Interpreting, 5/2, pp. $187-220$.

Ficchi, V. (I999). «Learning consecutive interpretation. An empirical study and an autonomous approach». Interpreting, 4/2, pp. 199-218.

Flavell, J. H. (200o). El desarrollo cognitivo. Trad. de M. J. Pozo y J. Ignacio. Nueva ed. rev. Madrid: Visor.

Galperin, P. I. (1992). «Stage-by-stage formation as a method of psychological investigation». Journal of Russian and East European Psychology, 30 (4), 69-80.

Gile, D. (1995). Basic Concepts and Models for Interpreter and Translator Training. Amsterdam/Filadelfia: John Benjamins.

161

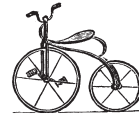




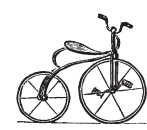

Hoffman, R. R. (I997a). «How can expertise be defined? Implications of research from cognitive psychology». Exploring Expertise. J. Fleck y R. Williams (eds). Edinburgh: University of Edinburgh Press, pp. 8I-Ioo.

162
(I997b). «The cognitive psychology of expertise and the domain of interpreting». Interpreting 2, I/2, pp. I89-230.

Kiraly, D. C. (200o). A Social Constructivist Approach to Translator Education. Empowerment from Theory to Practice. Manchester: St. Jerome.

Lantolf, J. P. (2000). Sociocultural Theory and Second Language Learning. Oxford/Nueva York: Oxford University Press.

Little, D. (1997). "Language Awareness and the Autonomous Language Learner». Language Awareness, 6, 2/3, pp. 93-I04.

Mackintosh, J. (I995). «A Review of Conference Interpretation: Practice and Training». Target, 7/I, pp. II9-I33.

Main, M. (1985). "Reflection and the Development of Learning Skills». En D. Boud et. al. (eds.). Reflection: Turning Experience into Learning. Londres: Kagan Page, pp. I92-213.

Monereo, C. (I995). «Enseñar a conciencia. ¿Hacia una didáctica metacognitiva?». Aula, 34, pp. 74-80.

Monereo, C. (coord.) et al. (1998). Estratègies d'Aprenentatge. Volum I: Assessorament i formació del professorat. Col.lecció Manuals. Barcelona: Edicions de la Universitat Oberta de Catalunya. Col.lecció Manuals.

Moser-Mercer, B. (2005a). «Challenges to Interpreter Training». 20 Jahre Transforum: Koordinierung von Praxis und Lehre des Dolmetschens und Übersetzens. Hildesheim: G. Olms, pp. 6I-72.

- (2005b). «The Teaching of Simultaneous Interpreting: The First 60 Years (1929-I989)». Forum, 3/I, pp. 205-225.

Moser-Mercer, B., U. H. Frauenfelder, B. Casado y A. Künzli (2000). «Searching to define expertise in interpreting», en K. Hyltenstam y B. EnglundDimitrova (eds.) Language processing and interpreting. Ámsterdam: John Benjamins, pp. I-2I.

Moser-Mercer, B.; B. Class y K. Seeber (2004). «Community building: Training interpreter trainers». Paper presented at the Fifth National
Conference on Interpreting Practice. Shangai.

Onrubia, J. (I993). «Enseñar: crear zonas de desarrollo próximo e intervenir». En C. Coll (ed.). El constructivismo en el aula. Barcelona: Graó, pp. IOI-I33.

Perry, N. E.; L. Phillips y L. R. Hutchinson (2006). «Preparing student teachers to support for selfregulated learning». Elementary School Journal, Io6, pp. $237^{-254}$.

Pöchhacker, F. (2004). Introducing Interpreting Studies. Londres: Routledge.

Pöchhacker, F. y M. Schlesinger (2002). The Interpreting Studies Reader. Londres/ Nueva York: Routledge.

Rampillon, U. (I997). «Be aware of awareness - oder Beware of awareness? Gedanken zur Metakognition im Fremdsprachenunterricht der Sekundarstufe I». En U. Rampillon y P. Zimmermann (eds). Strategien und Techniken beim Erwerb Fremder SprachenIsmaning: Hueber, pp. I73-I94.

Ribas, T. (2000). L'avaluació formativa en l'ensenyament $i$ l'aprenentatge de la composició escrita. Anàlisi de la incidència de la pauta d'avaluació en el procés de composició escrita. Tesis doctoral inédita. Universitat Autònoma de Barcelona.

Sainz, M. J. (1995). "Awareness and Responsibility: our Students as Partners». En C. Dollerup y V. Appel (eds.). Teaching Translation and Interpreting 3. Ámsterdam: John Benjamins, pp. I33-I43.

Seleskovitch, D. y M. Lederer (I989). Pédagogie raisonnée de l'interprétation. Bruxelles-Luxembourg: Didier érudition Opoce.

Van Lier, L. (1996). «Interaction in the Language Curriculum. Awareness, Autonomy and Authenticity». En C. N. Candlin (ed.). Applied Linguistics and Language Study. Nueva York: Longman, pp. 226-24I.

Vygotsky, L. S. (1978). Mind in Society. The Development of Higher Psychological Processes. Cambridge (Mass.): Harvard University Press.

Weber, W. (1989). «Improved Ways of Teaching Consecutive Interpretation». En L. Gran y J. Dodds (eds.). The Theoretical and Practical Aspects of Teaching Conference Interpretation. Udine: Campanotto Editore, pp. I6I-I67. 